\title{
Tracking objects out of the observer's view
}

\author{
Jiří Lukavský ${ }^{1}$, Lauri Oksama², Filip Děchtěrenko ${ }^{1}$ \\ ${ }^{1}$ Institute of Psychology, Czech Academy of Sciences, Prague, Czech Republic \\ ${ }^{2}$ Finnish Defence Research Agency, Human Performance Division, Järvenpää, Finland
}

Draft version, Feb 15, 2022. This paper has not been peer reviewed.

Please do not copy or cite without author's permission.

\section{Author note}

Jiří Lukavský (iD http://orcid.org/0000-0002-1082-229X

Lauri Oksama (D) https://orcid.org/0000-0001-7188-6396

Filip Děchtěrenko (iD https://orcid.org/0000-0003-0472-915X

Correspondence concerning this article should be addressed to Jiří Lukavský, Institute of Psychology, Czech Academy of Sciences, Hybernská 8, 11000 Prague, Czech Republic. Email: lukavsky@praha.psu.cas.cz 


\begin{abstract}
In everyday life, we often view objects through a limited aperture (e.g., soccer players on TV or cars slipping into your blind spot on a busy road), where objects often move out of view and reappear in a different place later. We modelled this situation in a series of multiple object tracking experiments, where we introduced a cover on the edges of the observed area and manipulated its width. This introduced systematic occlusions, which were longer than in previous MOT studies. Exp. $1(\mathrm{~N}=50)$ showed that tracking under such conditions is possible, although difficult. In Exp. $2(\mathrm{~N}=50)$ we manipulated the tracking workload and found that the performance improved in the most difficult condition with high amounts of cover. In Exp. 3 ( $N=50$ ), we asked the participants to keep track of objects' identities (MIT). Although MIT is subjectively more demanding, memorising identities improved performance in the most difficult conditions. Contrary to previous reports, we also found that even partial occlusions negatively affected tracking.
\end{abstract}




\section{Tracking objects out of the observer's view}

Life in a dynamic environment requires people deal with occlusions. Objects often move out of sight to reappear later. Although we cannot see occluded objects, we tend to experience them as persistent in space and time (Michotte, 1950). We represent the surface properties of objects (e.g., colour or shape) and use them together with spatiotemporal properties (proximity, expected location) to establish the object correspondence after the reappearance (Hollingworth \& Franconeri, 2009; Richard et al., 2008). The ability to represent and recover objects after disappearance develops early in our lives (Rosander \& von Hofsten, 2004; von Hofsten et al., 2007).

Representing and recovering a single object is not sufficient. In many everyday tasks, including crossing a street, driving or team sports, people must keep track of multiple moving objects simultaneously. A popular method for studying the underlying mechanisms is multiple object tracking (MOT; Pylyshyn \& Storm, 1988). The paradigm has been used and adapted to study attentional mechanisms, objecthood or individual differences, among others (Meyerhoff et al., 2017).

Previous experiments showed that people can track multiple objects during brief occlusions (Scholl \& Pylyshyn, 1999). When the occlusions were brief (approximately 300 $\mathrm{ms}$ ) and the objects reappeared close to the point of their disappearance $\left(1^{\circ}\right)$, the performance was comparable to that during the trials without occlusions. The participants were sensitive to the signals indicating occlusion (accretion/deletion led to better performance than gradual size changes or sudden teleportations). The occluder's visibility did not constitute a critical signal (occluders could be of the same colour as the background with no visible border). The participants could track objects when they disappeared behind their private occluders, which did not persist in the display or affect other objects (as if the objects dive/emerge under the display surface). With longer disappearance durations (up to $500 \mathrm{~ms}$ ) and faster object speeds, the performance gradually decreased (Horowitz et al., 2006).

Studying occlusion is often related to the questions of direction sensitivity and motion extrapolation. However, the ability to track occluded objects likely relies on simple proximity heuristics rather than extrapolations (Franconeri et al., 2012). Their experiments have shown that tracking performance is not affected by direction changes that occur when an object is occluded and that a larger distance between the occlusion entry/exit points makes the task more difficult (even if the exit trajectory corresponds with the entry trajectory). Analogously, shortening the distance between the entry/exit points of the occlusion makes the task easier (even if the exit trajectory is inconsistent with the entry trajectory). Probe detection experiments (Flombaum et al., 2008) show that people allocate more attentional resources to the areas where they expect occluded objects (both targets and distractors) relative to unoccluded objects or occluders with no objects hidden behind them. These increased attentional requirements might be indicative of the mechanisms waiting to recover the occluded object once it reappears. Interestingly, this effect also applies to distractors, which are actively inhibited during tracking when visible (Flombaum et al., 2008; Pylyshyn, 2006; Pylyshyn et al., 2008).

Although MOT is considered an attentional task (Huang et al., 2012), it is a complex task that often involves situations in which other mechanisms, such as memory or decision 
making, are required. It remains unclear how visual memory mechanisms contribute to MOT. Some of the theoretical models of MOT propose serial mechanisms that are involved in tracking (d'Avossa et al., 2006; Li et al., 2019; Oksama \& Hyönä, 2008). The existence of serial mechanisms presumes cooperation with a spatial memory component, which retains the locations of objects that are currently unattended. This is consistent with the observation that people report out-of-date object locations. Although the information in memory is periodically refreshed, it takes longer and leads to larger spatial errors, when more objects are being tracked (Howard \& Holcombe, 2008). However, parallel mechanisms sensitive to the tracking load might provide an alternative explanation (Howard et al., 2011).

People can engage memory when tracking. For example, people encode and use object properties such as colour if they help them distinguish targets and distractors during tracking and thus make the tracking easier (Makovski \& Jiang, 2009). When people are asked to remember identity information about individual targets (Horowitz et al., 2007; Multiple Identity Tracking or MIT; Oksama \& Hyönä, 2004), people can complete the task, but it is substantially more difficult. The tracking capacity in MIT is lower than that in standard location tracking. Horowitz et al. (2007) reported a drop in tracking capacity from 2.3-3.4 to 1.4-2.6 objects.

Dual-task experiments, when people are performing MOT while retaining information in visual working memory, also show a complex contribution of attention and memory in both tasks. Allen et al. (2006) showed that although spatial tapping (a visuospatial memory task) interferes with MOT performance, they observed larger interference when central executive processes were also required for the secondary task (as in the visual digit categorization task). Fougnie and Marois (2006) found that increasing the tracking workload in MOT has a detrimental effect on the number of objects stored in working memory. Although this suggests that both tasks share resources, the observed interference was smaller than in the dual-task condition with two working memory tasks. Importantly, people can concurrently remember the spatial positions of extra objects without detrimental effects on MOT performance (Howard et al., 2020).

Previous research focused only on brief occlusions (in hundreds of milliseconds). Here, we explore the human ability to track objects that are occluded longer (in seconds). In particular, we focus on situations, in which an object leaves the observer's view and it is more difficult to predict when and where the object will reappear. It is something we experience in everyday life. For example, when watching a sports match on TV, the camera captures only a part of the scene and individual players may enter or exit the view. This approach is also different from previous studies in which occluders were placed more centrally (Scholl \& Pylyshyn, 1999).

We designed a variant of the MOT task with large occluders on the edges of the display. In Experiment 1, we explored how the prolonged occlusion or unavailability of targets affected human tracking performance. In subsequent experiments, we manipulated the attentional and memory requirements of the task. This allowed us to measure their impact on the successful tracking of occluded objects. Contrary to dual-task experiments, our participants had a single goal and the task remained naturalistic and easy to understand. In particular, we manipulated the tracking workload in Experiment 2 and tested the effect of more attentional resources. In Experiment 3, the participants tracked the same number of targets, but they had to remember the targets' identities in half of the trials. 


\section{Experiment 1}

In previous MOT studies, the occlusion was very brief (hundreds of milliseconds). However, in real-world situations, people must cope with much longer disappearances of objects caused by occlusion or moving out of view. Experiment 1 aimed to explore the limits of the human capacity to track occluded objects. We presented the participants with a common MOT scenario with an aperture limiting their view. In other words, the participants tracked objects moving in a circular arena, and we manipulated the amount of cover in the proximity of the outer edges of the arena. In a within-subject design, we measured the tracking performance when up to $47 \%$ of the area was covered.

\section{Method}

\section{Participants}

Fifty participants from the Prolific platform participated in the study during August 2020. All participants self-reported normal or corrected-to-normal vision and no history of attention disorders. To increase data quality, we offered a performance bonus and a performance-based exclusion criterion. We offered the participants a $50 \%$ bonus if their performance was above the sample median. We excluded data from the participants whose performance in the two easiest conditions (pooled, no cover and 1-unit cover) could not be distinguished from guessing (99.9\% binomial distribution range). Originally, 51 people took part in the study, and we excluded data from one participant due to low performance. All reported experiments were approved by the Ethics Board of the Institute of Psychology, Academy of Sciences of the Czech Republic.

\section{Apparatus and stimuli}

Stimuli were presented online using a custom script built on the jsPsych framework (de Leeuw \& Motz, 2016). The experiment was presented via the participant's web browser. Participation was limited only to desktop/laptop users. We specified the size of the stimuli in pixels. To make the reading easier, we report sizes in arbitrary units $(1 \text { unit }=24 \text { pixels })^{1}$.

Tracking stimuli were eight black disks (1 unit diameter) presented on a mid-grey background (see Fig.1). The disks' movement was constrained by an invisible annulus (with a 12-unit outer radius and a 3-unit inner radius). The participants' view was limited to a circular aperture (radius 9 to 12 units). The edge of the aperture was marked with a thin black line (width 1 pixel). The aperture ensured that objects partly or completely disappeared when approaching within 1-3 units of the outer edge of the annulus.

\footnotetext{
${ }^{1}$ Given this scale, the entire display subtended $24 \times 24=576$ pixels, which would fit the browser area without vertical scrolling in all cases. One unit corresponds approximately to 5.5 to $7 \mathrm{~mm}$ in common displays.
} 


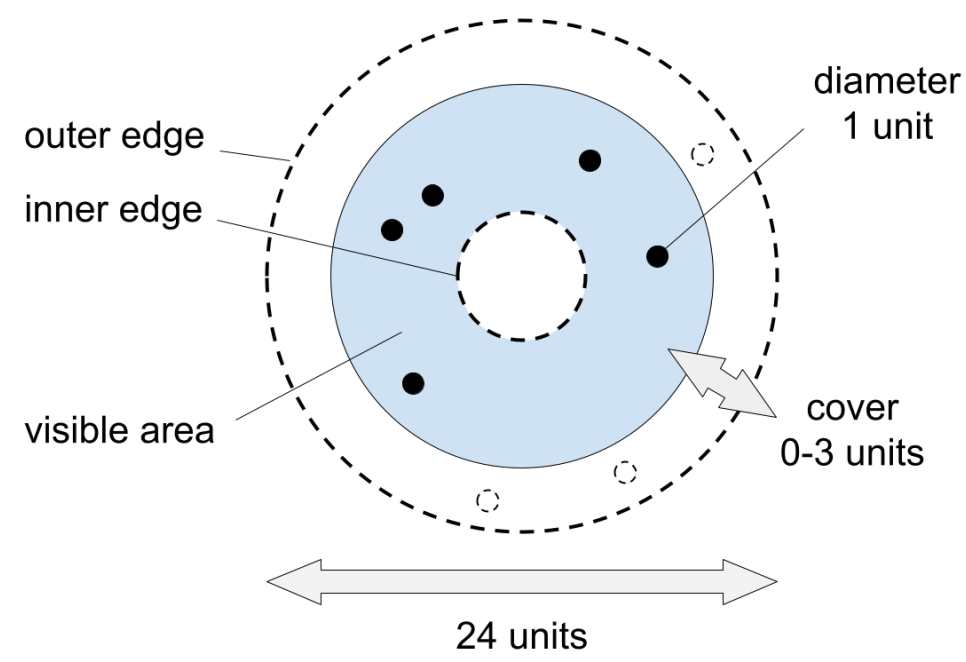

Fig. 1. Stimuli used in Exp.1. The objects are visible only within a blue area. Dashed lines represent edges where objects bounced. Note that both blue and white areas were painted midgrey in the experiment.

The disks moved at a fixed speed of 5 units per second on straight-line paths and randomly changed direction every 1.5-2.5 seconds. They also bounced off the inner and outer edge of the annulus and off each other. When bouncing off each other, the objects kept an interobject distance of 1 unit. We introduced the inner edge to route objects towards the outer edge and the areas hidden from the participants' view.

We pregenerated the objects' trajectories for 200 trials using the motrack package (Lukavsky, 2021) and randomly selected trials from this pool. The same pool of trajectories was used in the subsequent experiments. All materials, including stimuli, code and analytical scripts, are available at the OSF repository (https://osf.io/w52qx/).

\section{Design and procedure}

In Experiment 1, we manipulated the amount of space visible to the participants. The view was either not limited at all (aperture radius 12 units) or partly limited (radius 9, 10 or 11 units). Limiting the view created a band (width 3,2 or 1 unit) by the vicinity of the outer edge of the annulus, where the objects were partly or completely hidden. In each condition, the participants could see $53.3 \%, 67.4 \%, 83.0 \%$ or $100 \%$ of the area to which objects could move (area of the annulus).

At the beginning of the trial, a participant could see a circular arena and all eight objects. Four target objects were highlighted in light green for 2 seconds and then turned back to black. All objects started moving. After 8 seconds, all objects stopped, and those hidden behind the aperture boundary became visible. The participant selected the four targets with the mouse. Selected objects were marked with a rectangular frame, and it was possible to deselect an object and choose a different object. When four objects were selected, the response was automatically submitted, and the participant was given feedback (correct responses highlighted in green, incorrect in red). 
The experiment consisted of a training ( 4 trials, one for each condition) and the main part (52 trials, 13 per condition). The main part was presented in a single block with no breaks, but the participants could follow their own pace and take breaks if they needed. The experiment took approximately 20 minutes to complete.

\section{Analysis}

In our analysis, we express the tracking performance as the probability that a single target is correctly marked because it facilitates comparison when trials differ in the number of targets. This measure is different from common approaches, such as the number of correctly marked targets or tracking capacity (Hulleman, 2005). It resembles the proportion correct measure, although the analysis is based on logistic regression, not ANOVA. The observed probability of successful tracking $\left(p^{\prime}\right)$ is a combination of the true probability of successful tracking $(p)$ and the probability of lucky guessing $\left(p_{g}\right)$. Assuming $p^{\prime}=p+(1-p) \times p_{g}$, the true probability is calculated as $p=\left(p^{\prime}-p_{g}\right) /\left(1-p_{g}\right)$. The chosen adjustment assumes that people have no additional information about individual objects and that their guessing is uniformly distributed.

We used a Bayesian multilevel logistic model to estimate the probability of successful tracking. The model featured a single categorical predictor (cover condition, 4 levels) and group-level effects (intercepts and slopes per participant). The Bayesian analysis allowed us to further analyse and transform the posterior distributions. We transformed the results of the logistic regression back to the probability scale and adjusted the probability values for guessing. We report the results as $95 \%$ highest posterior density intervals. We used the brms $\mathrm{R}$ package to perform the analysis (Bürkner, 2017) with weakly informative priors. The model specification, including priors, can be found in the OSF repository.

\section{Results}

To illustrate the effect of cover, we took the pool of pregenerated trajectories used in the experiment and calculated distributions for a number of parameters, including the mean number of visible objects or the median duration of invisibility (see Fig. 2). With increasing cover size, fewer objects were visible at each moment, and naturally, objects were hidden for longer. Each object disappeared approximately 2-3x per trial, and this number was not dependent on the amount of cover. 
How many times

does each object disappear?

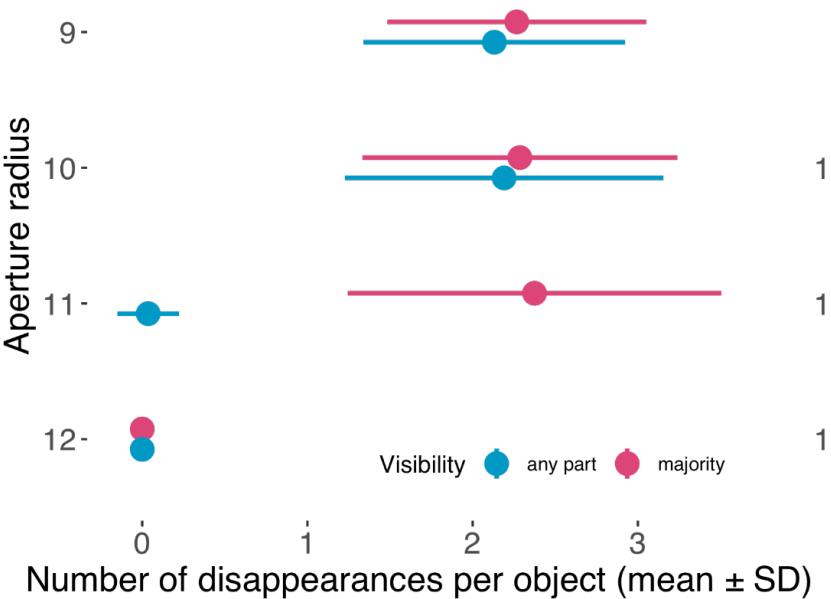

How many objects

are visible at each moment?

9 -

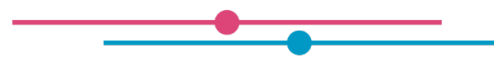

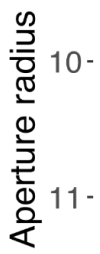

$12-$

$\begin{array}{ccccc}\dot{4} & \dot{5} & \dot{6} & \dot{7} & \dot{8} \\ \text { Number of simultaneously } & \text { visible objects } & (\text { mean } & \text { (SD) }\end{array}$

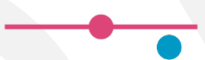

When object disappears, how long does it stay hidden?
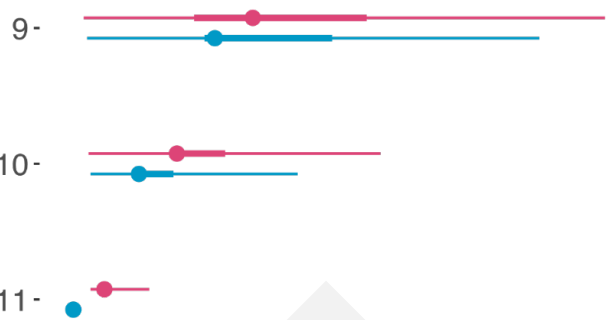

$12-$

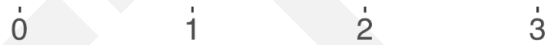

Duration of a single disappearance [s]

How much time

is an object hidden in total?

$9-$

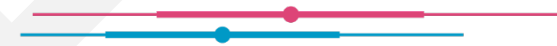

10

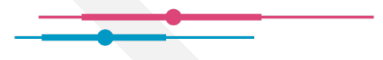

11 -

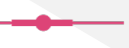

$12-$

ó

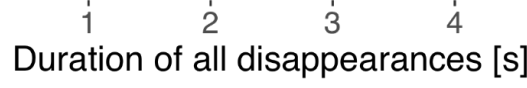

Fig. 2. Illustrative statistics for the stimuli used in our experiments reporting the number of disappearances behind cover, their durations and how many objects were visible at each moment as a function of aperture radius. Count data are expressed as the mean and standard deviations, and continuous data depict the mean and the $66 \%$ and $95 \%$ quantiles. Colour indicates different definitions of cover: "majority" (pink) means the object is considered visible if more than $50 \%$ of its area is visible; "any part" (teal) means the object is considered visible unless it disappears completely.

As expected, the performance decreased when larger parts of the arena were covered (see Fig. 3). Interestingly, the performance decreased in the condition with the smallest cover (aperture radius 11 units, covered area $17 \%$ ) by 5.5\% (95\% HPDI [1.2, 9.9]), where the objects could not be fully covered (the width of the cover was identical to the object width; thus, the objects could disappear only for very brief moments before bouncing back). Even in the most difficult condition, the performance was still above the chance level 
(43.3\%, 95\% HPDI [37.9; 48.7]). Note that the performance is already corrected for guessing; thus, the guessing level corresponds to $0 \%$.
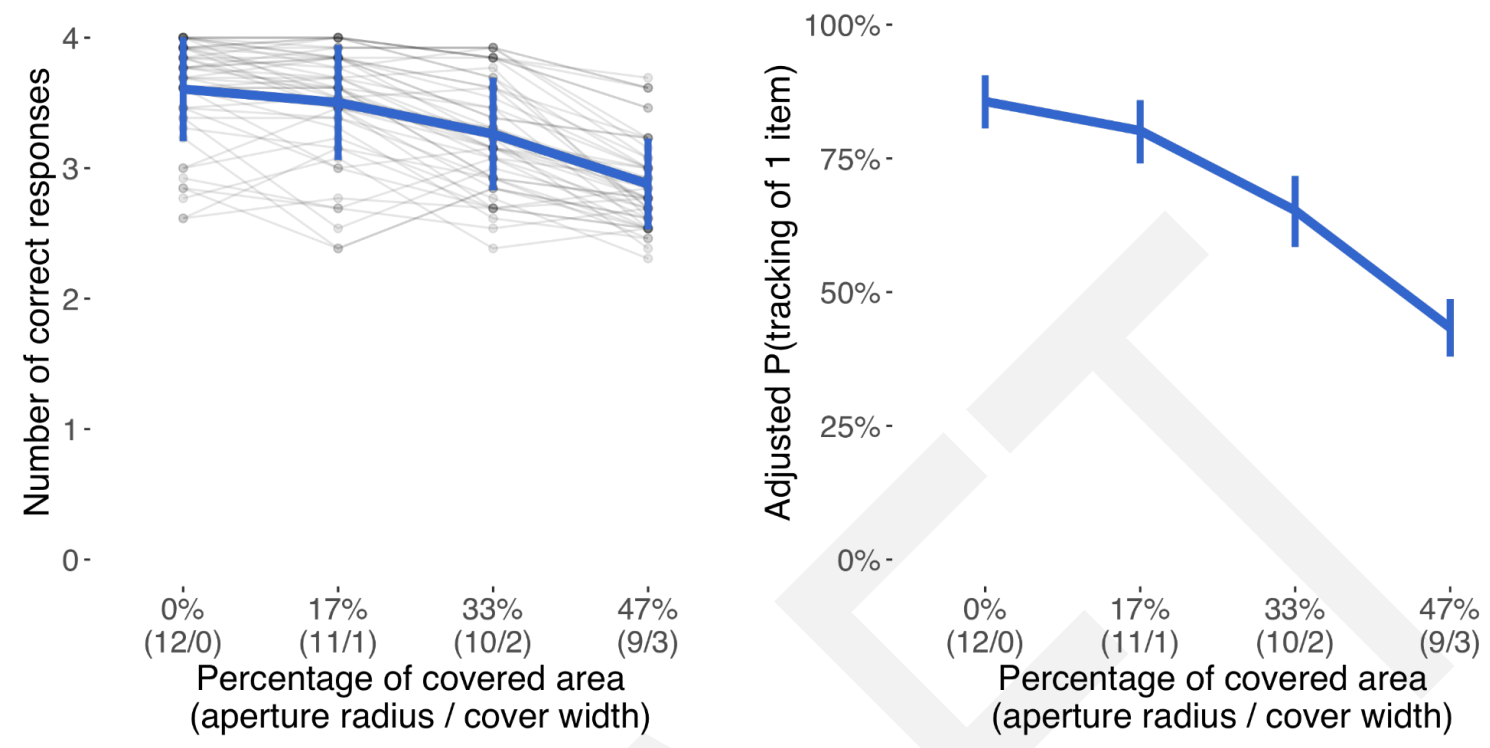

Fig. 3. The tracking performance as a function of cover. Left: The individual and the average number of correct responses (mean $\pm \mathrm{SD}$ ). Right: The performance is expressed as a probability of successfully tracking a single item (error bars denote 95\% HPDI). The cover is shown in percentages of the space where the objects could move.

\section{Discussion}

In Experiment 1, we introduced a cover causing prolonged or repeated occlusion of the tracked objects. Very often, the tracked objects were completely covered for 1-2 seconds, which is substantially longer than in previous studies. Although Scholl and Pylyshyn (1999) report occlusions of up to $2640 \mathrm{~ms}$, they explain that these were cases of incomplete occlusions during which objects "brushed" occluder boundaries. When we consider complete occlusions, Horowitz et al. (2006) used the longest disappearances of $507 \mathrm{~ms}$. The objects in the most difficult conditions of Franconeri et al. (2012) were completely hidden for approximately $600-1000$ ms (given the speed and the width of the occluder).

Our results show that the tracking performance is substantially impaired by the size of the cover. In the most difficult condition, where the cover subtended almost half of the area in which the objects could move, the participants' performance was significantly beyond the chance level (44\% after correction for guessing). This shows that people were able to track (or recover) objects beyond the aperture boundary.

Interestingly, the performance declined in the condition with the smallest cover (aperture radius 11 units) relative to the no-cover condition. In the 11-unit condition, the covered band spanned only 1 unit, i.e., the diameter of the tracked objects. Consequently, 
the objects could be completely covered for only brief moments before they bounced off the arena edge. Most of the time, they were completely visible or only partially covered. This contrasts with previous findings (Scholl \& Pylyshyn, 1999) that brief occlusions accompanied with accretion and deletion did not differ from unoccluded conditions. The role of accretion/deletion signals could be lower because occlusions often occurred in the periphery. Potentially, the presence of longer occlusions in other trials could evoke uncertainty in participants and contributed to the performance drop. Alternatively, this particular claim could be revised. Although the original study had proper power for detecting overall effects between conditions, the power for the contrast between the no occlusion and occlusion conditions was only $29 \%$ (for one-sided test). In subsequent experiments, we excluded the 12-unit condition and focused only on the conditions with some amount of cover.

\section{Experiment 2}

Experiment 1 showed that tracking occluded objects is very difficult but possible. In Experiment 2, we explored whether we can alleviate the task difficulty by reducing the tracking workload. We hypothesised that when the workload is lower and more resources are available, people might be better at tracking the occluded objects or at recovering them when they reappear. We independently manipulated the tracking workload (tracking 2 to 4 targets) and the amount of cover.

\section{Method}

\section{Participants}

Fifty students from Charles University in the Czech Republic participated in the experiment during November 2020. The students received course credit for their participation, and we offered no motivation bonus as in Exp. 1. To detect potential noncompliance, we compared the performance in trials with the largest visible area with a 99.9\% guessing threshold. Data from no participant were excluded from the analysis based on this performance check.

\section{Apparatus and stimuli}

The apparatus and stimuli were similar to those used in Experiment 1. Contrary to the previous experiment, we marked the outer edge of the annulus with a thin dark grey line (width 1 pixel) to provide information about the extent of nonvisible space in which objects could move.

\section{Design and procedure}

In Experiment 2, we manipulated two conditions. The first condition was the amount of space visible to the participants (aperture radius 9, 10 or 11 units). Compared to Experiment 1, we did not include the condition with no cover. The second condition was the cognitive workload, i.e., whether the participants were asked to track 2, 3 or 4 targets (4 targets tracked in Experiment 1). 
The experiment started with 9 training trials (one of each condition combination) followed by the main part with 117 trials (13 per condition). The participants were offered a short break after 40 and 80 trials. The experiment took approximately 40 minutes to complete.

\section{Analysis}

We used a Bayesian multilevel logistic model similar to the model used in Exp. 1. The probability of successful tracking was predicted from two predictors (cover: 3 levels, workload: continuous variable) and their interaction, and the model included group-level effects (intercepts and slopes per participant). We back-transformed the results of the logistic regression to the probability scale and reported the results as the $95 \%$ highest posterior density intervals. The probabilities were adjusted for guessing as in Exp. 1.

\section{Results}

The changes in cover had a larger impact on the performance than the changes in the tracking workload (see Fig. 4). We can take tracking four targets as a baseline for the description of the results (also corresponding to the Exp. 1 setup). Adding an extra unit of cover at the border had substantial effects on the tracking performance, which dropped by $16.3 \%$ (95\% HPDI [12.8; 20.0]) for the 10-vs. 11-unit radius conditions and further by another $22.2 \%$ (95\% HPDI [17.5; 27.0]) for the 9- vs. 10-unit radius conditions. The smaller tracking workload led to nonzero improvements in tracking but smaller than the effect of cover. The tracking workload benefits were higher with larger cover. Tracking one less target in the 11-unit radius condition yielded a $4.4 \%$ improvement (95\% HPDI [2.0; 7.5]) but a larger $10.3 \%$ improvement $(95 \%$ HPDI $[6.5 ; 14.8])$ in the 9-unit radius condition.

Note that the tracking workload results are normalised, i.e., expressed as the probability of successfully tracking a single target.

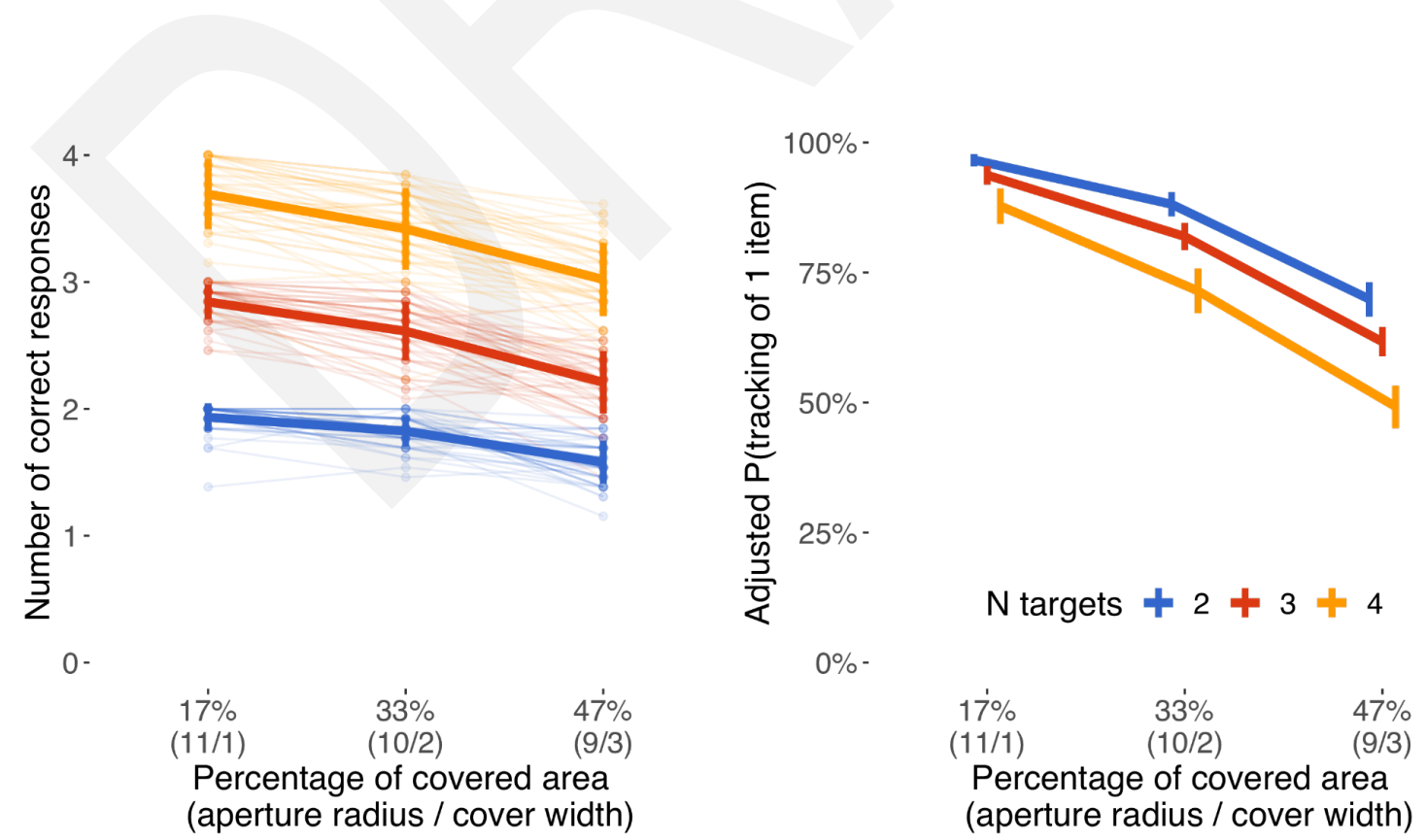


Fig. 4. The tracking performance as a function of cover and tracking workload. Left: The individual and the average number of correct responses (mean \pm SD). Right: The performance is expressed as a probability of successfully tracking a single item (error bars denote $95 \% \mathrm{HPDI}$ ). The cover is shown in percentages of the space in which the objects could move.

Additionally, we compared the performances in Experiments 1 and 2. We repeated the analysis on a joint dataset containing data from the conditions present in both experiments (trials featuring 4 targets and the cover between 9 and 11 units). After backtransforming the results to the percentage scale and correcting for guessing, we found that the performance in Exp. 2 was slightly higher, but the differences between the experiments were not convincing. The performance in Exp. 2 was higher by $2.0 \%$ (95\% HPDI [-0.2; +4.3]) in the easier condition (11-unit radius) and $6.0 \%$ (95\% HPDI $[-0.5 ;+12.4])$ in the more difficult condition (9-unit radius).

\section{Discussion}

Consistent with Exp. 1, we found that the tracking performance is substantially impaired by the size of the cover. The performance decline associated with the amount of cover was larger than the changes associated with the differences in tracking workload. The general performance was comparable in both experiments despite small differences.

Experiment 2 was longer, and different participants were recruited (students vs. Prolific) and featured a thin dark grey line to mark the extent of the nonvisible space in which objects could move.

The participants tracked the objects better when tracking only a smaller set of targets, which is not surprising and corresponds to the previous findings (Oksama \& Hyönä, 2004). The differences in tracking workload conditions look small, which could be attributed to two factors. First, we report normalised probabilities of successful tracking, and consequently, the differences are on a smaller scale. Second, the differences are more pronounced after adjusting for guessing, which varies across the workload conditions. Tracking only two targets could be easy, but if one target is lost, it is more difficult to recover it with a correct guess. Losing a target in a common MOT task may be rare, but this may happen more often in a challenging setup with occluders.

After correcting for guessing, we confirmed a small performance benefit of reduced workload. The benefit was present in all cover conditions and gradually increased with larger covers. This suggests that having more attentional resources provides more help in more difficult conditions that involve critical situations (e.g., cover, occlusions or collisions).

\section{Experiment 3}

In MOT, people must track the locations of a selected subset of objects, but they do not have to encode any additional information about them. If we ask them to remember the individual identities of the targets, the task becomes substantially more difficult. Identity tracking (MIT; Oksama \& Hyönä, 2004) leads to different gaze patterns in tracking (Hyönä et 
al., 2019), and additional cortical circuits related to object recognition and working memory are involved (Nummenmaa et al., 2017).

In the preceding experiments, the participants were asked to remember that there was one or a few targets in the covered area, so they could recover them when they reentered the arena. We hypothesised that additional cognitive resources required to track identities helped maintain the memory of the hidden targets and recover them later. In other words, the cover would affect the performance more in MOT and less in MIT.

\section{Method}

\section{Participants}

Fifty students from Charles University in the Czech Republic participated in the experiment during February 2021. The students received course credit for their participation, and we offered no motivational bonus as in Exp. 1. Data from an additional two participants were excluded (one of them failed the performance check, and both made very long breaks with a resulting experiment duration of over 2 hours).

\section{Apparatus and stimuli}

The apparatus and stimuli were similar to those used in Experiment 2. The workload was fixed, and the participants were asked to track 3 of the 8 objects. In MIT trials, the targets were marked with different colours (orange \#EDA98F, magenta \#DEA4EA, cyan \#4DC8DD) and with different glyphs (duck, butterfly, rabbit) from Auckland Optotypes (Hamm et al., 2018). The targets and distractors looked identical during the tracking phase (black with no glyphs).

\section{Design and procedure}

In Experiment 3, we explored the effect of cover (3 levels of aperture radius: 9, 10 and 11 units) and demands associated with identity tracking (MOT vs. MIT trials).

In MIT trials, we asked the participants to select objects of a given identity in a predefined order. We showed a picture cue (e.g., orange duck) in the top left corner outside of the annulus in which the objects moved. After the participants selected the object, which presumably corresponded to this cue, another cue appeared. The order of cues was randomised across the sample but fixed for each participant. During feedback, the correct locations of each object were shown, and the incorrect responses were marked red.

The experiment started with 12 training trials (two for each condition combination) followed by the main part with 120 trials (20 per condition). The participants were offered a short break after 40 and 80 trials. The experiment took approximately 40 minutes to complete.

\section{Analysis}

We used a Bayesian multilevel logistic model similar to the models used in previous experiments. The probability of successful tracking was predicted from two predictors (cover: 3 levels, task: MOT/MIT) and their interaction, and the model included group-level effects 
(intercepts and slopes per participant). We back-transformed the results of the logistic regression to the probability scale and reported the results as the $95 \%$ highest posterior density intervals. The guessing levels were $37.5 \%$ in MOT (tracking 3 of 8 objects) and $12.5 \%$ in MIT (1 object of 8 ).

\section{Results}

Although the numbers of correct responses for MOT and MIT were similar and the performance in MOT was higher by a very small margin (see Fig. 5 left), the conditions differed in their guessing levels. After correcting for guessing, most differences disappeared. In the 11 -unit radius condition, the performance in MOT was higher by $0.7 \%[-0.9 ; 2.3]$ and in 10 -unit radius condition by less than $0.1 \%[-2.5 ; 2.6]$. Therefore, our experiment does not provide evidence for better performance in MOT. In contrast, our data suggest that the difference after normalising for tracking a single item and correcting for guessing is within $3 \%$. Such difference is smaller than the difference caused by reducing the tracking workload by one item in Exp. 2. In the most difficult 9-unit radius condition, the MIT performance exceeded MOT by $9.4 \%$ [5.9; 12.9]. This shows that the additional cognitive resources invested in identity tracking facilitate the tracking behind cover or recovering objects when they are lost.

Consistent with our previous experiments, the amount of cover had a substantial effect on the tracking performance. For example, the performance in MOT dropped from an initial $92.3 \%[90.1 ; 94.4]$ to $81.9 \%[78.7 ; 84.9]$ and further to $57.7 \%[53.9 ; 61.6]$.

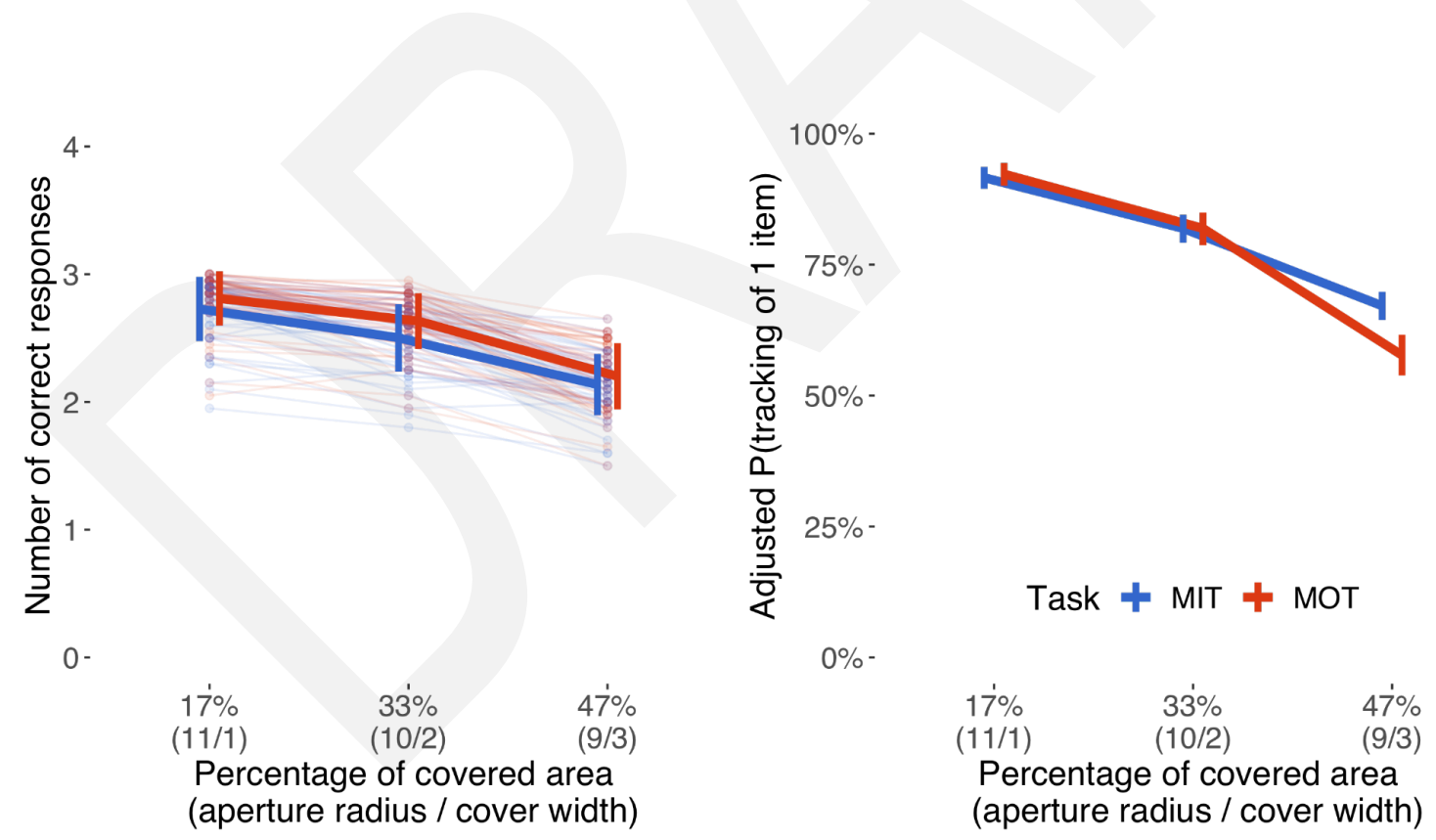

Fig. 5. The tracking performance as a function of cover and task (location vs identity tracking). Left: The individual and the average number of correct responses (mean $\pm S D$ ). Right: The performance is expressed as a probability of successfully tracking a single item (error bars denote 95\% HPDI). The cover is shown in percentages of the space in which the objects could move. 


\section{Discussion}

Although the number of correct responses in identity tracking was lower, the result must be interpreted carefully because the guessing levels differ across tasks. In standard MOT without occlusions, the difference in guessing levels may play a smaller role because people can track the assigned targets. Identity tracking under standard conditions requires extra effort, which may stress participants. When long-term occlusions are introduced, the task becomes more difficult, people may lower their ambitions, and identity tracking is not perceived as requiring as much effort.

The results support our initial hypothesis that the additional cognitive resources employed in identity tracking help overcome the effects of situations in which the tracked object becomes covered. The results are consistent with those of Exp. 2 that a reduced tracking workload improved performance only when the objects were covered.

\section{General discussion}

In a series of three experiments, we explored how people track objects during prolonged occlusions. The occlusions were longer than in previous experiments (seconds vs. hundreds of milliseconds) and resembled the situation in which people observe moving objects through a limited aperture (e.g., cars slipping into your blind spot on a busy road). Our experiments yielded three main findings.

First, people can track objects that disappear for longer durations (in seconds, see Fig. 2 for descriptive statistics about occlusions during a trial). In the most difficult condition, when almost half of the tracking area was covered, the participants still had an approximately $40-60 \%$ chance of successful tracking (after accounting for guessing). The durations of complete occlusions were longer than those in previous studies, where they reached 500 to $1000 \mathrm{~ms}$ in the most difficult cases (Franconeri et al., 2012; Horowitz et al., 2006).

Second, manipulating the attentional and memory workload in Experiments 2 and 3 affected the tracking of objects behind the cover. At the same time, the attentional and memory manipulations yielded small to negligible effects in conditions with small cover when the objects were visible most of the time. We found that when people track a smaller set of targets, they are less likely to lose them while they are covered. If people track objects using a fixed number of attentional indices or foci (Alvarez \& Cavanagh, 2005; Pylyshyn \& Storm, 1988), each object is associated with a single index or focus, and tracking a smaller set of objects should not yield better performance. Common strategies for correcting for guessing based on tracking capacity (Horowitz et al., 2007; Hulleman, 2005) share these assumptions: the targets within the tracking capacity limit are tracked with $100 \%$ efficiency, and guessing applies only to the objects tracked beyond the tracking limit. The very difficult tracking tasks, such as those in the present study, dispute the assumption of $100 \%$ efficiency. It makes sense to assume that people can flexibly allocate attentional resources (Alvarez \& Franconeri, 2007) to improve their tracking and to overcome critical situations in tracking (e.g., collisions or cover). Experiment 3 showed that engaging memory (asking people to remember the identities of targets) also led to improved performance in the conditions with large covers. 
Third, the tracking performance decreased even for the smallest cover condition in Experiment 1. In other words, the cover affected performance even when objects are only rarely occluded in their totality. This condition featured a 1-unit band, which could cover the size of an object, but the object immediately bounced back. This may have evoked uncertainty in the participants, but it could not lead to target-distractor confusion behind the cover. Previous experiments with brief occlusions reported unimpaired performance in accretion/deletion signal condition relative to the no occlusion condition (Scholl \& Pylyshyn, 1999). The contradictory results may be a consequence of the different positions of occluders. Here, the occluder was placed further in the periphery and served as an aperture boundary. In this setup, the occlusions were often observed in the participants' peripheral vision and/or subject to crowding. This could have weakened the importance of the accretion/deletion signal, and even very brief disappearances could be viewed as disruptive. Alternatively, even partial occlusions affect tracking.

People can deal with long-term occlusions in the periphery on different levels. We speculate that there are three potential strategies to help people successfully track objects during prolonged occlusions (object recovery, area monitoring, or recovery facilitation). First, all occlusions are treated alike regardless of their duration (object recovery). People employ mechanisms similar to those employed for brief occlusions (Teichmann et al., 2021), relying on motion information and extrapolating objects' future locations or alternatively relying on proximity cues (Franconeri et al., 2012; Horowitz et al., 2006; Scholl \& Pylyshyn, 1999).

Alternatively, when a target disappears, people can stop tracking the target, reallocate their resources and focus the location-based attention on the area at which they expect it to reappear (area monitoring). Simultaneously, they are still tracking the remaining targets. This is consistent with the results of previously described probe detection experiments (Flombaum et al., 2008).

Finally, people can stop tracking the occluded object and rely on conscious strategies to recover it later (recovery facilitation). Instead of constantly monitoring the area at which the object is expected to reappear, they may remember its specific position (e.g., "the only remote object on the right") and recover it at a convenient opportunity later (during the tracking or response phase). Previous experiments showed that people can update the set of tracked objects, i.e., stop tracking an old target and start tracking a distractor while maintaining all other targets (Wolfe et al., 2007).

Our experiments did not identify the strategies the participants used. Nevertheless, our data show that people can track objects occluded for longer durations, suggesting a potential role of high-level strategies (area monitoring or recovery facilitation).

An important finding is the disappearance of differences in tracking accuracy between MIT and MOT tasks when the guessing is corrected for. This finding is not limited to our data. For example, when we inspected the results of another study comparing MOT and MIT with hidden identities (Wu \& Wolfe, 2018) and corrected the accuracy for guessing, the overall accuracy was higher in MIT than in MOT. From the participant's perspective, MIT with hidden identities feels more demanding. This might be a result of its dual-task nature, as people in addition to attentional tracking must memorise and retain extra information about the objects. However, those demands do not need to be reflected in decreased performance, and potentially, the engagement of memory might help individuals overcome 
challenging tracking situations. Potentially, the presence of cover could encourage participants in MIT trials. When an experiment features only MOT and MIT trials, people realise that MIT is more demanding and may hesitate to exert more effort because they think it is beyond their limits. The cover manipulations made the experiment substantially more demanding, and the subjective difference in MOT and MIT might be diminished.

To facilitate comparison with other MOT studies, we highlight some of the distinguishing features. As noted earlier, the occluders were placed in the periphery and limited the participants' view. The width of the occluder was marked with a thin line in Experiments 2 and 3. In Experiment 1, the width of the occluder was not immediately apparent but could be learned over the course of the experiment because the outer edge was always placed at a 12-unit radius. The objects' motion was predictable to a large extent: they moved at constant speeds in straight lines, randomly changing directions approximately every 2 seconds (or bounced off the edges or other objects). We placed an invisible circle in the centre of the arena to repel the objects from the central parts towards the occluder. We wanted to avoid situations in which an object moves around the centre of the display, bounces off other objects and rarely approaches the occluder. The resulting motion patterns were balanced with a small bias towards being visible (e.g., when $47 \%$ of the arena was covered, the objects were hidden for $35 \%$ of the time).

In a series of three experiments, we demonstrated that people can track objects that are repeatedly covered for prolonged durations, although the tracking performance is substantially affected. Additional attentional resources (low workload) or additional memory instruction (memorising identities) improved the performance mainly in the conditions with a high amount of cover. Contrary to previous reports, we found that even partial occlusions negatively affected tracking. Our data also suggest that the apparent lower performance in MIT than in MOT might be a result of more difficult guessing because it is less likely for a guess to be correct in MIT. We speculate that people rely on additional processes to recover hidden objects when they reappear. These strategies are important in the difficult variants of MOT, but are likely also involved in traditional MOT variants.

\section{Declaration of conflicting interests}

The authors declared no potential conflicts of interest with respect to the research, authorship, and/or publication of this article.

\section{Funding}

The author(s) disclosed receipt of the following financial support for the research, authorship, and/or publication of this article: This work was supported by the Czech Science Foundation (Grant GA 19-07690S) and by the Academy of Finland (\#325693). 


\section{References}

Allen, R., Mcgeorge, P., Pearson, D. G., \& Milne, A. (2006). Multiple-target tracking: A role for working memory? The Quarterly Journal of Experimental Psychology, 59(6), 11011116. https://doi.org/10.1080/02724980543000097

Alvarez, G. A., \& Cavanagh, P. (2005). Independent resources for attentional tracking in the left and right visual hemifields. Psychological Science, 16(8), 637-643. https://doi.org/10.1111/j.1467-9280.2005.01587.x

Alvarez, G. A., \& Franconeri, S. L. (2007). How many objects can you track? Evidence for a resource-limited attentive tracking mechanism. Journal of Vision, 7(13). https://doi.org/10.1167/7.13.14

Bürkner, P.-C. (2017). brms: An R Package for Bayesian Multilevel Models Using Stan. Journal of Statistical Software, 80(1), 1-28. https://doi.org/10.18637/jss.v080.i01

d'Avossa, G., Shulman, G. L., Snyder, A. Z., \& Corbetta, M. (2006). Attentional selection of moving objects by a serial process. Vision Research, 46(20), 3403-3412. https://doi.org/10.1016/j.visres.2006.04.018

de Leeuw, J. R., \& Motz, B. A. (2016). Psychophysics in a Web browser? Comparing response times collected with JavaScript and Psychophysics Toolbox in a visual search task. Behavior Research Methods, 48(1), 1-12. https://doi.org/10.3758/s13428-0150567-2

Flombaum, J. I., Scholl, B. J., \& Pylyshyn, Z. W. (2008). Attentional resources in visual tracking through occlusion: the high-beams effect. Cognition, 107(3), 904-931. https://doi.org/10.1016/j.cognition.2007.12.015

Fougnie, D., \& Marois, R. (2006). Distinct capacity limits for attention and working memory: Evidence from attentive tracking and visual working memory paradigms. Psychological Science, 17(6), 526-534. https://doi.org/10.1111/j.1467-9280.2006.01739.x

Franconeri, S. L., Pylyshyn, Z. W., \& Scholl, B. J. (2012). A simple proximity heuristic allows tracking of multiple objects through occlusion. Attention, Perception \& Psychophysics, 74(4), 691-702. https://doi.org/10.3758/s13414-011-0265-9

Hamm, L. M., Yeoman, J. P., Anstice, N., \& Dakin, S. C. (2018). The Auckland Optotypes: An open-access pictogram set for measuring recognition acuity. Journal of Vision, 18(3), 13-13. https://doi.org/10.1167/18.3.13

Hollingworth, A., \& Franconeri, S. L. (2009). Object correspondence across brief occlusion is established on the basis of both spatiotemporal and surface feature cues. Cognition, 113(2), 150-166. https://doi.org/10.1016/j.cognition.2009.08.004

Horowitz, T. S., Birnkrant, R. S., Fencsik, D. E., Tran, L., \& Wolfe, J. M. (2006). How do we track invisible objects? Psychonomic Bulletin \& Review, 13(3), 516-523. https://doi.org/10.3758/BF03193879

Horowitz, T. S., Klieger, S. B., Fencsik, D. E., Yang, K. K., Alvarez, G. A., \& Wolfe, J. M. (2007). Tracking unique objects. Perception \& Psychophysics, 69(2), 172-184. https://doi.org/10.3758/BF03193740

Howard, C. J., \& Holcombe, A. O. (2008). Tracking the changing features of multiple objects: Progressively poorer perceptual precision and progressively greater perceptual lag. Vision Research, 48(9), 1164-1180. https://doi.org/10.1016/j.visres.2008.01.023

Howard, C. J., Masom, D., \& Holcombe, A. O. (2011). Position representations lag behind targets in multiple object tracking. Vision Research, 51(17), 1907-1919.

https://doi.org/10.1016/j.visres.2011.07.001

Howard, C. J., Pole, R., Montgomery, P., Woodward, A., Guest, D., Standen, B., Kent, C., \& 
Crowe, E. M. (2020). Visual spatial attention and spatial working memory do not draw on shared capacity-limited core processes. Quarterly Journal of Experimental Psychology , 73(5), 799-818. https://doi.org/10.1177/1747021819897882

Huang, L., Mo, L., \& Li, Y. (2012). Measuring the interrelations among multiple paradigms of visual attention: an individual differences approach. Journal of Experimental

Psychology. Human Perception and Performance, 38(2), 414-428. https://doi.org/10.1037/a0026314

Hulleman, J. (2005). The mathematics of multiple object tracking: From proportions correct to number of objects tracked. Vision Research, 45(17), 2298-2309. https://doi.org/10.1016/j.visres.2005.02.016

Hyönä, J., Li, J., \& Oksama, L. (2019). Eye Behavior During Multiple Object Tracking and Multiple Identity Tracking. Vision Research, 3(3), 37. https://doi.org/10.3390/vision3030037

Li, J., Oksama, L., \& Hyönä, J. (2019). Model of Multiple Identity Tracking (MOMIT) 2.0: Resolving the serial vs. parallel controversy in tracking. Cognition, 182, 260-274. https://doi.org/10.1016/j.cognition.2018.10.016

Lukavsky, J. (2021). motrack: Tools for Multiple Object Tracking experiments (Version 0.3.0) [Computer software]. https://github.com/jirilukavsky/motrack

Makovski, T., \& Jiang, Y. V. (2009). Feature binding in attentive tracking of distinct objects. Visual Cognition, 17(1-2), 180-194. https://doi.org/10.1080/13506280802211334

Meyerhoff, H. S., Papenmeier, F., \& Huff, M. (2017). Studying visual attention using the multiple object tracking paradigm: A tutorial review. Attention, Perception \& Psychophysics, 1-20. https://doi.org/10.3758/s13414-017-1338-1

Michotte, A. (1950). A propos de la permanence phénoménale faits et theories. Acta Psychologica, 7, 298-322. https://doi.org/10.1016/0001-6918(50)90021-7

Nummenmaa, L., Oksama, L., Glerean, E., \& Hyönä, J. (2017). Cortical Circuit for Binding Object Identity and Location During Multiple-Object Tracking. Cerebral Cortex , 27(1), 162-172. https://doi.org/10.1093/cercor/bhw380

Oksama, L., \& Hyönä, J. (2004). Is multiple object tracking carried out automatically by an early vision mechanism independent of higher-order cognition? An individual difference approach. Visual Cognition, 11(5), 631-671. https://doi.org/10.1080/13506280344000473

Oksama, L., \& Hyönä, J. (2008). Dynamic binding of identity and location information: A serial model of multiple identity tracking. Cognitive Psychology, 56(4), 237-283. https://doi.org/10.1016/j.cogpsych.2007.03.001

Pylyshyn, Z. W. (2006). Some puzzling findings in multiple object tracking (MOT): II. Inhibition of moving nontargets. Visual Cognition, 14(2), 175-198. https://doi.org/10.1080/13506280544000200

Pylyshyn, Z. W., Haladjian, H. H., King, C. E., \& Reilly, J. E. (2008). Selective nontarget inhibition in Multiple Object Tracking. Visual Cognition, 16(8), 1011-1021. https://doi.org/10.1080/13506280802247486

Pylyshyn, Z. W., \& Storm, R. W. (1988). Tracking multiple independent targets: evidence for a parallel tracking mechanism. Spatial Vision, 3(3), 179-197. https://doi.org/10.1163/156856888X00122

Richard, A. M., Luck, S. J., \& Hollingworth, A. (2008). Establishing object correspondence across eye movements: Flexible use of spatiotemporal and surface feature information. Cognition, 109(1), 66-88. https://doi.org/10.1016/j.cognition.2008.07.004

Rosander, K., \& von Hofsten, C. (2004). Infants' emerging ability to represent occluded 
object motion. Cognition, 91(1), 1-22. https://doi.org/10.1016/s0010-0277(03)00166-5

Scholl, B. J., \& Pylyshyn, Z. W. (1999). Tracking Multiple Items Through Occlusion: Clues to Visual Objecthood,. Cognitive Psychology, 38(2), 259-290.

https://doi.org/10.1006/cogp.1998.0698

Teichmann, L., Edwards, G., \& Baker, C. I. (2021). Resolving visual motion through perceptual gaps. Trends in Cognitive Sciences, 25(11), 978-991. https://doi.org/10.1016/j.tics.2021.07.017

von Hofsten, C., Kochukhova, O., \& Rosander, K. (2007). Predictive tracking over occlusions by 4-month-old infants. Developmental Science, 10(5), 625-640. https://doi.org/10.1111/j.1467-7687.2007.00604.x

Wolfe, J. M., Place, S. S., \& Horowitz, T. S. (2007). Multiple object juggling: changing what is tracked during extended multiple object tracking. Psychonomic Bulletin \& Review, 14(2), 344-349. https://doi.org/10.3758/bf03194075

Wu, C.-C., \& Wolfe, J. M. (2018). Comparing eye movements during position tracking and identity tracking: No evidence for separate systems. Attention, Perception \& Psychophysics, 80(2), 453-460. https://doi.org/10.3758/s13414-017-1447-x 